\title{
BMJ Open Hearing and vision screening tools for long-term care residents with dementia: protocol for a scoping review
}

\author{
Katherine S McGilton, ${ }^{1,2}$ Fiona Höbler, ${ }^{1,3}$ Jennifer Campos, ${ }^{1,4}$ Kate Dupuis, ${ }^{4,5}$ \\ Tammy Labreche, ${ }^{6}$ Dawn M Guthrie, ${ }^{7}$ Jonathan Jarry, ${ }^{8}$ Gurjit Singh, ${ }^{1,3,9,10}$ \\ Walter Wittich ${ }^{8,11,12,13}$
}

To cite: McGilton KS, Höbler F, Campos J, et al. Hearing and vision screening tools for long-term care residents with dementia: protocol for a scoping review. BMJ Open 2016;6:e011945. doi:10.1136/bmjopen-2016011945

- Prepublication history and additional material is available. To view please visit the journal (http://dx.doi.org/ 10.1136/bmjopen-2016011945).

Received 16 March 2016 Revised 24 May 2016 Accepted 30 June 2016

CrossMark

For numbered affiliations see end of article.

Correspondence to Dr Katherine S McGilton; kathy.mcgilton@uhn.ca

\section{ABSTRACT}

Introduction: Hearing and vision loss among longterm care (LTC) residents with dementia frequently goes unnoticed and untreated. Despite negative consequences for these residents, there is little information available about their sensory abilities and care assessments and practices seldom take these abilities or accessibility needs into account. Without adequate knowledge regarding such sensory loss, it is difficult for LTC staff to determine the level of an individual's residual basic competence for communication and independent functioning. We will conduct a scoping review to identify the screening measures used in research and clinical contexts that test hearing and vision in adults aged over 65 years with dementia, aiming to: (1) provide an overview of hearing and vision screening in older adults with dementia; and (2) evaluate the sensibility of the screening tools.

Methods and analysis: This scoping review will be conducted using the framework by Arksey and O'Malley and furthered by methodological enhancements from cited researchers. We will conduct electronic database searches in CENTRAL, CINAHL, EMBASE, MEDLINE and PsycINFO. We will also carry out a 'grey literature' search for studies or materials not formally published, both online and through interview discussions with healthcare professionals and research clinicians working in the field. Our aim is to find new and existing hearing and vision screening measures used in research and by clinical professionals of optometry and audiology. Abstracts will be independently reviewed twice for acceptance by a multidisciplinary team of researchers and research clinicians.

Ethics and dissemination: This review will inform health professionals working with this growing population. With the review findings, we aim to develop a toolkit and an algorithmic process to select the most appropriate hearing and vision screening assessments for LTC residents with dementia that will facilitate accurate testing and can inform care planning, thereby improving residents' quality of life.

\section{INTRODUCTION}

Dementia affects a person's ability to understand explanations, follow directions or

\section{Strengths and limitations of this study}

- This scoping review takes a rigorous and systematic approach to a broad research question that brings together two traditionally stand-alone areas of research and clinical practice by professionals working in both fields, to answer the critical issue of how to most effectively screen both hearing and vision abilities in older adults with dementia, residing in a long-term care setting.

- We will include all published literature with original research data from electronic databases and online search engines, in any language and within any setting that has measured hearing and/or vision in older adults with any form of dementia, whether for research or clinical purposes.

- This review will describe the psychometric properties of assessments found in the literature and those used in the field, and evaluate the acceptance and feasibility of their use with this population.

- A limitation of this scoping review may lie in the large scale of its aggregate findings for vision or hearing measures with populations who have cognitive impairment, and, for reasons of feasibility, we may not be able to provide a more in-depth quality analysis of the individual studies reported therein.

correctly interpret interpersonal communication. ${ }^{1}$ Indeed, language impairment is often seen as one of the first symptoms of dementia. $^{23}$ The dementias, particularly in their moderate to severe staging, are characterised by deficits in memory and language processing attributed to the temporal lobe area, and are reflected in the individual's ability to recognise, generate and repeat words, organise information in conversation, as well as variable impairments of grammatical, semantic (related to meaning) and lexical (vocabulary) knowledge. ${ }^{4-6}$ These problems can have profound implications for effective interactions in longterm care (LTC) facilities. The prevalence of dementia is $58 \%$ among residents in this setting, ${ }^{7}$ and there are increasing incidence 
rates of this disease in a rapidly ageing population. ${ }^{8} 9$ When residents cannot articulate their needs or cannot be understood because of their dementia, they frequently become frustrated or agitated. Furthermore, LTC staff may not correctly attribute these behaviours to various causes, and often underestimate the prevalence of sensory loss and its effects on communication. ${ }^{10}$

The challenges of the resulting communication difficulties in residents with dementia are compounded by hearing and vision problems that progress as people age. ${ }^{4}$ Sensory loss is widespread among older adults, and is often overlooked in those living in residential settings. Nursing home residents tend to be older and have higher levels and more severe physical and cognitive impairment than those living in the community. ${ }^{11}$

Hearing loss is the third most prevalent chronic condition in older adults, ${ }^{12}$ estimated in up to $50 \%$ of those aged over 65 years. ${ }^{13}$ Vision impairment (low vision with visual acuity $<20 / 70$ ) is reported in $18 \%$ of individuals aged 70 years or over. ${ }^{14}$ Dual sensory loss or deafblindness was found to have the highest prevalence in older adults in LTC settings, at $\sim 25 \%$, compared to those noninstitutionalised or dwelling in other settings. ${ }^{15}$ Notably, the prevalence of such sensory loss among adults with dementia has been shown to be higher than in those who are cognitively intact, ${ }^{16}{ }^{17}$ with hearing loss alone found in more than $90 \%$ of cognitively impaired patients. ${ }^{18}$

Although the mechanisms underlying the association between cognitive and sensory impairment remain unknown, it has been suggested that this relationship may result from a common neuropathological origin in the brain underlying both sensory loss and cognitive decline, effects of social isolation caused by both sensory and cognitive loss, and/or increased cognitive/attentional load caused by sensory loss. ${ }^{19} 20$

This evidence suggests significant increases in the prevalence of, and association between, sensory and cognitive decline in a population of older adults (above 65 years) that is rapidly increasing. ${ }^{21}{ }^{22}$ Unsurprisingly, the incidence of sensory impairment is higher in the LTC setting, with $\sim 80 \%$ of LTC residents experiencing at minimum mild hearing loss and $\sim 50 \%$ having a moderate to severe impairment. ${ }^{23-25}$ Visual impairment is twice as high in LTC residents as among the general population of the same age, with a reported prevalence of between $30 \%$ and $57 \% .{ }^{26-29}$ Ultimately, hearing and/or vision loss is found in two-thirds of residents and dual sensory loss affects one-third of LTC residents. ${ }^{26}$

For these reasons, there is a pressing need to further investigate the relationship between hearing, vision and cognitive impairment, as well as to develop appropriate interdisciplinary interventions to moderate their effects on older and vulnerable persons, by employing a comprehensive interdisciplinary and collaborative approach. ${ }^{30}{ }^{31}$ We will adopt this approach in our search for sensitive hearing and vision screening tools that appropriately identify sensory impairment as the first step in this rehabilitation process.

\section{Pilot project in training Resident Centred Communication}

In a recent pilot project funded by the Alzheimer Society of Canada, our research team investigated the effects of enhancing interactions between staff and LTC residents through training in a Resident Centered Communication Intervention (RCCI) (McGilton KS, Pichora-Fuller MK, Shaw A, et al. In preparation. Can we assist staff to communicate more effectively with persons with dementia living in Long Term Care Homes?). This study took place in a 128-bed, for-profit, LTC home in Ontario, Canada with 12 residents who had a diagnosis of dementia and 20 caregiving staff. The aim of the study was to determine if a Resident Communication Centred Intervention could influence caregiver and residents' outcomes. The RCCI involved a dementia-care workshop, the development of individualised resident communication care plans by a speech-language pathologist, with staff supported at the bedside by an advanced practice nurse to implement the care plans. Individualised communication care plans were tailored according to the cognitive, sensory and linguistic abilities of the residents.

In order to address concerns raised in the literature and by LTC staff, sensory assessments, as well as linguistic and cognitive testing, was undertaken to better understand each resident's current linguistic and cognitive abilities from the outset. ${ }^{32-34}$ Two major problems were identified:

1. Usual screening in LTC facilities: Only residents who are flagged by staff or family members as possibly having sensory problems are assessed by the relevant specialist. At other times, identification of sensory problems may arise through the standardised resident assessment (known as the MDS 2.0), which includes items on hearing and vision, and is completed at admission, quarterly and with a change in the clinical status of the resident. ${ }^{35}$ Nonetheless, these assessments rely heavily on observation and reporting, are not comprehensive and have been shown to be frequently inadequate in identifying those in need of specialist referral. ${ }^{36}$

2. Standard test procedures: The tests used to supplement information about hearing and vision acquired from patient records have been validated in a noncognitively impaired population. There are no equivalent vision screening tests designed specifically for persons with dementia in LTC facilities, and no best practice protocols for audiological examination of this population. ${ }^{37}$ Not surprisingly, in our pilot study, there was limited success in administering standardised tests (eg, audiogram assessment and Functional Linguistic Communication Inventory), as residents often had difficulty following instructions. 
For example, the following adaptations were made to the standardised testing procedures for hearing and vision: (1) audiometric testing: two participants were unwilling to complete the test and some were unable to learn to respond consistently to pure-tone stimuli; thus, live voice testing at a conversational level was improvised using simple tasks; (2) vision testing: participants often demonstrated difficulty following test instructions and maintaining prolonged attention even though instructions were communicated using clear and simple speaking skills. ${ }^{38}$ Therefore, when necessary, test procedures were modified; for example, for patients who had difficulty sustaining attention, only a subset of the Teller cards was shown (MK Pichora-Fuller, A Shaw, M Saragosa, et al. In preparation. Screening assessments for persons with dementia: trials and tribulations and lessons learnt).

In the traditional research domains of vision and hearing, participants with severe cognitive impairment are often excluded from recruitment and data collection, as tests that are otherwise standardised in their administration would need to be adapted for this population. For example, the requirement of reading letters on an eye chart relies on the ability to identify and remember these letters, and then repeat them, making these test formats unsuitable for individuals with impaired memory and language abilities, thus having to be substituted with the spelling of familiar words (such as the person's name) or basic numbers chart. This resulting exclusion process results in the limited scope of recent publications on the topic of sensory and comorbid cognitive loss.

Although previous literature reviews have been carried out in the area of dual sensory loss or deafblindness, reporting on its frequency and effects on functionality, ${ }^{39}$ comorbidities and impact on older adults above 65 years, ${ }^{40}$ as well as the sensory impact on dementia care, ${ }^{41}$ vision related quality of life in residents with dementia, ${ }^{42}$ and hearing loss with cognitive impairment, ${ }^{4}{ }^{43}$ a comprehensive review of hearing and vision assessments and their clinical utility in this growing population is not yet available. Researchers as well as health service providers in the field of deafblindness agree that the co-presentation of vision and hearing loss is not simply additive but multiplicative, thereby creating a new and more complex type of sensory impairment. ${ }^{44-46}$

This scoping review aims to address the need for adaptable and standardised screening by identifying suitable and validated hearing and vision measures for persons with dementia that can be used in LTC residencies. The main objective of our larger study is to address the reported problems of identifying adults in LTC facilities who are in need of referral to a hearing or vision specialist for second-level assessment, and thereby enable more specialised care and treatment for hearing and vision loss in this vulnerable population, which in turn will serve to promote their participation and engagement, and improve quality of care.

\section{Methods and analysis}

\section{Methodology}

Our scoping team of reviewers will be multidisciplinary, comprising clinician-scientists ${ }^{\mathrm{i}}$, researchers and clinicians specialising in the fields of nursing, audiology, optometry, cognitive and perceptual psychology, clinical neuropsychology and speech-language pathology. Consistent with the broad scope of our areas of interest, we will adopt the methodological framework set out by Arksey and O'Malley, ${ }^{47}$ employing a scoping approach to review the existing literature and to examine the extent, range and nature of research activity, identify research gaps in this literature, and then summarise and disseminate research findings, as outlined in online supplementary appendix 1 .

We will further adhere to the methodological enhancements based on previously published scoping reviews by providing transparency, reproducibility and utility with the presentation of this protocol. ${ }^{48}$ We aim for consistency in labelling and definition of scoping terms ${ }^{49}$ and maintaining a broad search strategy with clearly defined concepts and their continuous refinement. ${ }^{50}$ Additionally, we will use multidisciplinary expertise and group consultation within the scoping team to inform and guide the definition of the search criteria and clinical applicability of data for extraction, ${ }^{50} 51$ and to allow for post hoc development of inclusion/exclusion criteria and data synthesis in terms of the value yielded by qualitative or quantitative analysis of results. ${ }^{48}$

We will also conduct interviews with front-line practitioners and LTC staff to add more information, meaning and applicability to our search results. Finally, we will provide a summary of the current research activity and possible clinical implications of the evidence to further clinical research, practice and policy. ${ }^{50}$ Encompassing the fields of hearing and vision, we bring together two stand-alone concepts using established guidelines ${ }^{49}$ to form the research question: which hearing and vision screening measures and practices are effective in identifying hearing and vision impairments in older adults with dementia? Our aims are to: (1) provide an overview of the use of hearing and/or vision screening tools in persons with dementia; and (2) evaluate the sensibility of these measures.

It has been argued that despite having evidenced reliability, validity and responsiveness to change, instruments can be underused due to numerous reasons including their practicality, ${ }^{53}$ and therefore evaluating an instrument's sensibility (which includes face and content validity) should be an important first step to see if this will be

${ }^{\mathrm{i}}$ For the purpose of our study, we consider researchers to be those individuals whose primary training is focused on research methodologies, techniques and skills to conduct research (eg, $\mathrm{PhD})$; whereas we consider practitioners as those individuals whose primary focus during their training was the acquisition of skills for the purpose of delivering a clinical service (eg, RSW). We acknowledge that, in the case of clinician-scientists, there is a certain overlap between these two categories, likely beneficial to our purposes. We aim to have representation of all three groups on our team. 
acceptable in the research or clinical field. In this sense, sensibility should also be assessed before ecological validity, as completing the test successfully and acceptably with the intended population is most indicative of its feasibility, rather than real-world validity and applicability of results. The reliability and validity of the tools selected with consideration of sensibility will be carried out at a later stage of the process in developing the screening package. Sensibility will be defined as the feasibility and acceptability of an instrument in a specified assessment context. $^{54}$ Finally, our scoping results will then be reported in partitioned reviews dedicated to: (1) hearing; (2) vision; and (3) dual sensory assessment.

\section{Search methods}

In order to investigate the extent of screening literature available and to identify any gaps in research and clinical practice, we are defining screening measures as objective tests and instruments appropriate for use in the preliminary evaluation of hearing and/or visual ability (eg, hearing threshold or visual acuity). These screening instruments are not necessarily used for the diagnosis of a hearing or vision problem, but rather a reliable, valid and sensitive tool for detecting when further evaluation is warranted by a hearing or vision specialist. ${ }^{55}$ A broad definition of screening methods will be adopted that include paper-based tests, as well as technologies involving software solutions in the form of apps for mobile devices and higher-tech devices such as portable ophthalmic or audiometric equipment.

Electronic database searches will be conducted in CENTRAL, CINAHL, EMBASE, MEDLINE and PsycINFO by an Information Specialist at the Toronto Rehabilitation Institute-University Health Network. This will be augmented by web-based grey literature searches, for published and unpublished data in books or journals, including conference proceedings and abstracts, dissertations or theses, project reports and government documents, and test searches using Google Scholar and Opengrey, and the instrumental database for Health and Psychosocial Instruments). Trialled key search terms appropriate to each database will be used, with more narrow definitions of the terms used in the grey literature search as listed in online supplementary appendix 2 .

The publication years will be limited to between 1995 and 2016, without any language restrictions applied, to capture the full variation of possible tests being used. Search results will be filtered with removal of duplicates. Both peer reviewed and non-peer reviewed publications will be considered, including quantitative and qualitative research articles, assessment and treatment studies, as well as conference proceedings and academic dissertations that involve the reporting of original data.

Further to the grey literature search of online databases, information on available assessments and published research will also be gathered through interviews with front-line LTC staff and experts in optometry and audiology, and used to identify screening methods currently employed in the field, ultimately enriching the scoping results. Ten optometrists and audiologists, along with a convenience sample of 20 front-line nursing staff working in LTC with residents who have dementia, and those who are responsible for completing the MDS 2.0 (including sensory screening items), will be invited to participate in these interviews. The information collected from environmental scan interviews will be analysed by means of thematic content analysis to identify the important points regarding screening approaches and materials used by healthcare professionals. These professionals will be interviewed by a member of the research team and asked about: (1) their experiences of working with persons who have dementia, as well as hearing and vision loss; (2) how they identify which residents have sensory impairments; (3) ways in which sensory screening could be improved; and (4) which key elements should be included in a screening package. This will also mark the first step in continuous engagement and consultation with hearing and vision specialists throughout this study. ${ }^{50}$

\section{Study selection}

Consistent with recommendations by Levac et $a l,{ }^{50}$ the selection of studies: (1) will involve searching the literature, refining the search strategy based on the scope of the initial results and judged feasibility of reviewing all articles for study inclusion; (2) will require the scoping team to convene by teleconference at the beginning of this process to discuss decisions surrounding study inclusion and exclusion, for a second time after a trial run of the search strategy for possible refinement of procedures, and after all reviews have been completed for discussion of the full process; and (3) will employ at least two reviewers to independently read and rate each abstract for possible inclusion, with final arbitration by a third reviewer if consensus is not reached.

The resulting studies will be screened by this team of reviewers based on the title and abstract. Two reviewers will independently make a decision to exclude articles from the review based on the agreed on exclusion criteria, which are listed in online supplementary appendix 2.

Further, in line with guidelines by Levac et $a l^{50}$ each scoping team member will be briefed by an advance conference call on the exclusion criteria, their rationale and the coding system, providing the facility to raise any concerns and offer clarification where needed. Exclusion criteria and coding procedures will be trialled by reviewers on the first 50 citations before the team reconvenes by phone to discuss and resolve any issues with the coding guidelines, with the objective of reaching $100 \%$ consensus on the scoring procedures. Our scoping team will have expertise in areas of gerontology, vision, hearing or cognitive evaluation; however, examples of hearing and vision assessments will be provided to reviewers as reference points prior to the review process to facilitate clarification where expertise is not 
established, for example, the ETDRS Chart ${ }^{56}$ or Cardiff Acuity Test for visual acuity, or Otoacoustic emissions and pure-tone audiometry for hearing abilities.

Finally, as with the screening of abstracts, the full articles will be reviewed by two independent reviewers to confirm inclusion, with disagreements arbitrated by a third reviewer. ${ }^{50}$ Nevertheless, this process will remain iterative with each step continuously assessed for feasibility based on the search results and the analytical resources available within the scoping team.

\section{Data extraction}

After being approved for inclusion, research data will be extracted independently by two reviewers, with final confirmation by a third reviewer. ${ }^{47}$ The data collection form will be developed in collaboration with and following the approval of all members of the scoping team and charted in a standardised Excel spreadsheet, again approved by all collaborators. All reviewers will be provided with a sample extraction therein to guide them through this process. The spreadsheet will chart relevant data that will attempt to answer the research questions, as well as satisfy study objectives for the evaluation of test sensibility, ${ }^{48}$ while also providing information required by content experts in the final stage of the consultation exercise, including:

- Authors

- Year of publication

- Country

- Testing environment (eg, clinic, research laboratory, care home, LTC facility)

- Study design

- Sampling method

- Participant demographics (age, gender, dementia type, comorbidities)

- Name of tool

- Areas of testing (vision or hearing)

- Testing duration (time required to complete)

- Successful completion of test (including number of incomplete tests)

- Adaptations made for this clinical population (instructions provided)

- Measurement outcomes

- Interpretation of results (use of assessment data)

- Reported psychometric properties (validity, reliability, sensitivity, specificity, positive predictive values)

- Integrity of administration (who administered test, who interpreted the data).

\section{Data analysis}

Owing to the broad scope of our review question, we predict the generation of a large data set of resulting studies that use assessments of hearing, vision or both. We will therefore take a descriptive approach in providing a quantitative summary of the research findings, ${ }^{57} 58$ outlining the extent of research utility documented for each hearing and/or vision assessment in persons with dementia. These data will then be used to evaluate and summarise the sensibility of each instrument.
Sensibility is defined by Feinstein ${ }^{59}$ in terms of comprehensibility, replicability, suitability, ease of use, face/ construct validity, content validity and scale of purpose; and refers to an instrument's feasibility (or efficiency of purpose) in a specified screening context and its acceptability (of content and interpretation) to its intended users. $^{54}$ The evaluation of sensibility is an important first step before looking at the reliability, validity and responsiveness of an instrument, ${ }^{53} 54$ and critical to its acceptance and actual utility with the intended population. ${ }^{60}$

Once the review data have been summarised and grouped by the measures used, the charted information will be evaluated by the scoping team using a devised scoring sheet to rate the instrument's sensibility. Reviewers will score each test on a seven-point rating scale ( $1=$ strongly disagree, $7=$ strongly agree) to rate its qualities in terms of: (1) appropriateness; (2) objectivity; (3) content; and (4) discriminative power. ${ }^{53}$ The screening tool will be considered sensible if a mean score equal to or above five has been rated on at least $80 \%$ of the questionnaire items, and if none of the questionnaire items receive a mean rating of $3{ }^{54}$

\section{Consultation}

To add rigour and ensure the continued involvement of stakeholders throughout the process, we are engaging in a consultation exercise with clinical and research experts in hearing, vision and dual sensory impairment. ${ }^{47} 5051$ We have aligned our review process with this recommendation and will apply a multidimensional consultative approach in: (1) employing a multidisciplinary scoping team in the main review process; (2) engaging in investigative discussion with front-line professionals in the form of environmental scan interviews; and in (3) reporting our review findings to an expert panel of content developers, who will carry out the development of a hearing and vision screening package with devised guidelines as part of a later stage in this larger project.

We will organise a meeting with this expert panel to discuss the results of the current review, and with these findings employ a consensus method of modified RAND/UCLA Appropriateness Method, RAM, (RAND, research and development corporation; UCLA, University of California, Los Angeles) and Delphi method ${ }^{6162}$ to evaluate and help select the tests and procedures to be included in the screening package. Our panel will comprise of experts with specialised clinical and/or research experience in the fields of clinical neuropsychology, nursing, geriatrics, audiology, optometry and software development, recruited from the professional networks of the members of the study team. Given the highly specialised nature of this field of research, the network of specialists is tightly knit and many of the pertinent players are known to each other, making this identification and recruitment process relatively speedy. These experts will identify tests and procedures which they believe could be administered by front-line staff in LTC in a reasonable time period based on the strength of evidence provided 
by the results from the literature review and environmental scan interviews. A workflow of this first phase is charted in online supplementary appendix 3 .

Immediately following the development of the package of sensory screening tools, a similar consensus method will also be used to guide the development of a scoring mechanism (if adaptation of a screening tool for this population requires modification of the original scoring instructions) and decision-making process to identify which assessments to use with a specific resident and the criteria for referral to a relevant specialist. The panel members will reconvene in a final meeting to re-evaluate the screening tools in practice and make their final recommendations.

Future recommendations may also include the best approaches staff can use to conduct the screening assessments to acquire the most representative data possible. A noteworthy concern for this population is the reported variability in the symptomatic manifestation of Alzheimer's disease and mixed dementia, in terms of 'good days' associated with improved cognition and functioning and 'bad days' involving poor memory and increased agitation. ${ }^{63}$ Such implications for assessment will be outlined in the narrative contextualisation of search results.

\section{ETHICS AND DISSEMINATION}

The scoping review protocol presented in this paper will identify and describe the feasibility and acceptability of hearing and vision screening tools used with persons who have dementia, in research as well as in the clinical domain. We have chosen to use a scoping review methodology to allow for inclusion of all types of studies and policies that have targeted screening of the older adult. The review will summarise the available evidence in what has been done to screen for hearing and vision loss, as well as the sensitivity and sensibility of these tools in diagnosing this type of sensory impairment. Healthcare professionals have little guidance on how to assess for hearing and vision concerns in this population; thus, a comprehensive review of screening techniques will be a valuable resource and is a next important step for healthcare providers working in the primary healthcare setting. This type of review also allows us to include consultations with key stakeholders to identify gaps in the evidence and research that need to be addressed in future investigations.

Timely screening of hearing and vision will: (1) facilitate identifying those in need of referral to a hearing or vision professional; (2) enable individually tailored care for residents, thus promoting the health and well-being of older adults with dementia by enabling this population to participate more fully in programmes and care activities offered in LTC; (3) identify sensory impairments and, by addressing them to whatever extent possible, care plans can be adapted to accommodate for these impairments; and (4) allow tests of cognitive function that factor in sensory loss to be used, thus yielding a much more accurate gauge of residents' true levels of dementia. ${ }^{32}$
With these review findings, we envision a package of tools and a process for tool selection that considers the degree and nature of hearing and vision sensory loss. The anticipated result of this larger project will include: (1) recommendations for screening hearing in LTC facilities; (2) recommendations for screening vision in LTC facilities; (3) instructions for administering each screening tool and generating a score; (4) instructions for interpreting these scores; and (5) a description of the psychometric properties of the tools including reliability and validity; to facilitate the accurate screening of hearing and vision in older adults with dementia living in LTC, resulting in better personalised care, and thus possibly contributing to improvements in social participation, clinical interaction and overall quality of life for these residents. ${ }^{64}$

\section{Author affiliations}

${ }^{1}$ Department of Research, Toronto Rehabilitation Institute-University Health Network, Toronto, Ontario, Canada

${ }^{2}$ Lawrence S. Bloomberg Faculty of Nursing, University of Toronto, Ontario, Canada

${ }^{3}$ Department of Speech-Language Pathology, University of Toronto, Toronto, Ontario, Canada

${ }^{4}$ Department of Psychology, University of Toronto, Toronto, Ontario, Canada ${ }^{5}$ Department of Audiology, Baycrest Health Sciences, Toronto, Ontario, Canada

${ }^{6}$ Centre for Sight Enhancement, School of Optometry and Vision Science, University of Waterloo, Waterloo, Ontario, Canada

${ }^{7}$ Department of Kinesiology \& Physical Education and Health Sciences,

Faculty of Science, Wilfrid Laurier University, Ontario, Canada

${ }^{8}$ School of Optometry, University of Montréal, Montréal, Quebec, Canada

${ }^{9}$ Phonak AG, Stäfa, Switzerland

${ }^{10}$ Department of Psychology, Ryerson University, Toronto, Ontario, Canada

${ }^{11}$ School of Physical and Occupational Therapy, McGill University, Montréal,

Quebec, Canada

${ }^{12} \mathrm{CRIR} /$ Centre de réadaptation MAB-Mackay du CIUSSS du Centre-Ouest-del'île-de-Montréal. Montréal, Quebec, Canada

${ }^{13} \mathrm{CRIR} /$ Institut Nazareth et Louis-Braille du CISSS de la Montérégie-Centre, Montréal, Quebec, Canada

Contributors KSMcG is responsible for project conception and along with $\mathrm{FH}$ and WW for writing the protocol. JC, KD, TL, DMG, JJ and GS were involved in editing and revising the protocol.

Funding This work was supported by the Alzheimer Society Research Programme (ASRP), Alzheimer Society of Canada; grant number RG 16-08.

\section{Competing interests None declared.}

Ethics approval Ethical approval was granted by the Research Ethic Boards of the University Health Network and Baycrest Health Sciences for environmental scan interviews with LTC front-line staff.

Provenance and peer review Not commissioned; externally peer reviewed.

Open Access This is an Open Access article distributed in accordance with the Creative Commons Attribution Non Commercial (CC BY-NC 4.0) license, which permits others to distribute, remix, adapt, build upon this work noncommercially, and license their derivative works on different terms, provided the original work is properly cited and the use is non-commercial. See: http:// creativecommons.org/licenses/by-nc/4.0/

\section{REFERENCES}

1. Kim ES, Bayles KA. Communication in late-stage Alzheimer's disease: relation to functional markers of disease severity. Alzheimer's Care Q 2007;8:43-52.

2. Klimova B, Maresova $P$, Valis M, et al. Alzheimer's disease and language impairments: social intervention and medical treatment Clin Interv Aging 2015;10:1401-7. 
3. Tang-Wai DF, Graham NL. Assessment of language function in dementia. Geriatr Aging 2008;11:103-10.

4. Pichora-Fuller MK, Dupuis K, Reed M, et al. Helping older people with cognitive decline communicate: hearing aids as part of a broader rehabilitation approach. Semin Hear 2013;34:308-30.

5. Mansur LL. Language alterations in alzheimer's disease and other dementias. Language disturbances in adulthood: New advances from the neurolinguistics perspective. Dubai, UAE: Bentham Science Publishers Ltd. 2011:59-70.

6. Vuorinen E, Laine M, Rinne J. Common pattern of language impairment in vascular dementia and in Alzheimer disease. Alzheimer Dis Assoc Disord 2000;14:81-6.

7. Seitz D, Purandare N, Conn D. Prevalence of psychiatric disorders among older adults in long-term care homes: a systematic review. Int Psychogeriatr 2010;22:1025-39.

8. Prince M, Wimo A, Guerchet M, et al. Alzheimer's disease international (ADI). World Alzheimer Report 2015: the global impact of dementia. An analysis of prevalence, incidence, cost \& trends. London, UK: Alzheimer's Disease International (ADI), 2015. http:// www.alz.co.uk/research/WorldAlzheimerReport2015.pdf

9. Alzheimer Society of Canada. A new way of looking at the impact of dementia in Canada. 2012. http://www.alzheimer.ca/ /media/ Files/national/Media-releases/asc_factsheet_new_data_09272012 en.pd

10. Burnip LG, Erber NP. Staff knowledge regarding hearing loss and communication among nursing home residents. Aust J Ageing 1997;16:40-3.

11. Carpenter I, Hirdes JP. Using interRAI assessment systems to measure and maintain quality of long-term care. In: OECD \& European Commission (Eds). A Good Life in Old Age? Monitoring and Improving Quality in Long-term Care. Paris: OECD Publishing, 2013;93-159.

12. Yueh B, Shapiro N, MacLean $\mathrm{CH}$, et al. Screening and management of adult hearing loss in primary care: scientific review. J Am Med Association 2003;289:1976-85.

13. Cruickshanks KJ, Wiley TL, Tweed TS, et al. Prevalence of hearing loss in older adults in Beaver Dam, Wisconsin the epidemiology of hearing loss study. Am J Epidemiol 1998;148:879-86.

14. Crews JE, Campbell VA. Vision impairment and hearing loss among community-dwelling older Americans: implications for health and functioning. Am J Public Health 2004;94:823-9.

15. Vaal J, Gussekloo J, de Klerk MM, et al. [Combined vision and hearing impairment: in an estimated $30,000-35,000$ people aged 55 years or over in the Netherlands]. Ned Tijdschr Geneeskd 2007;151:1459-63.

16. Uhlmann RF, Larson EB, Koepsell TD, et al. Visual impairment and cognitive dysfunction in Alzheimer's disease. J Gen Intern Med 1991;6:126-32.

17. Uhlmann RF, Larson EB, Rees TS, et al. Relationship of hearing impairment to dementia and cognitive dysfunction in older adults. JAMA 1989;261:1916-19.

18. Gold M, Lightfoot LA, Hnath-Chisolm T. Hearing loss in a memory disorders clinic: a specially vulnerable population. Arch Neurol 1996;53:922-8.

19. Albers MW, Gilmore GC, Kaye J, et al. At the interface of sensory and motor dysfunctions and Alzheimer's disease. Alzheimers Dement 2015;11:70-98.

20. Lin FR, Yaffe $\mathrm{K}, \mathrm{Xia} \mathrm{J}$, et al. Hearing loss and cognitive decline in older adults. JAMA Intern Med 2013;173:293-9.

21. Ortman JM, Velkoff VA, Hogan $\mathrm{H}$. An Aging nation: the older population in the United States, current population reports, P25-1140. U.S. Census Bureau, Washington DC, USA, 2014. https://www.census.gov/prod/2014pubs/p25-1140.pdf.

22. Statistics Canada. The Canadian Population in 2011: age and sex Age and Sex, Census, 2011. Minister of Industry. Catalogue no. 98-311-X2011001, 2012. http://www12.statcan.gc.ca/ census-recensement/2011/as-sa/98-311-x/98-311-x2011001-eng.pdf

23. Weinstein BE. Geriatric audiology. New York: Thieme, 2000

24. Garahan MB, Waller JA, Houghton M, et al. Hearing loss prevalence and management in nursing home residents. J Am Geriatr Soc 1992;40:130-4.

25. Schow RL, Nerbonne MA. Hearing levels among elderly nursing home residents. J Speech Hear Disord 1980;45:124-32.

26. Yamada $\mathrm{Y}$, Vlachova M, Richter T, et al. Prevalence and correlates of hearing and visual impairments in European Nursing Homes: results from the SHELTER study. J Am Med Dir Assoc 2014;15:738-43. Elsevier Ltd

27. Owsley C, McGwin G, Scilley K, et al. The visual status of older persons residing in nursing homes. Arch Ophthalmol 2007;125:925-30.
28. Tielsch JM, Javitt JC, Coleman A, et al. The prevalence of blindness and visual impairment among nursing home residents in Baltimore. N Engl J Med 1995;332:1205-9.

29. Woodruff ME, Pack G. A survey of the prevalence of vision defects and ocular anomalies in 43 Ontario residential and nursing homes. Can J Public Health 1980;71:413-23.

30. O'Malley PG. Evolving insights about the impact of sensory deficits in the elderly. JAMA Intern Med 2013;173:299.

31. Swenor BK, Ramulu PY, Willis JR, et al. Research letters: the prevalence of concurrent hearing and vision impairment in the United States. JAMA Intern Med 2013;173:312-13.

32. Dupuis K, Pichora-Fuller MK, Chasteen AL, et al. Effects of hearing and vision impairments on the Montreal Cognitive Assessment. Neuropsychol Dev Cogn B Aging Neuropsychol Cogn 2015;22:413-37.

33. Wittich W, Murphy C, Mulrooney D. An adapted adult day centre for older adults with sensory impairment. Br J Vis Impairment 2014;32:249-62.

34. Weinstein BE, Arnsel L. Hearing loss and senile dementia in the institutionalized elderly. Clin Gerontologist 1986;4:3-15.

35. InterRAI. Instruments: long-term care facilities. 2016. http://www. interrai.org/long-term-care-facilities.html

36. Swanson MW, McGwin G Jr, Elliott AF, et al. The nursing home minimum data set for vision and its association with visual acuity and contrast sensitivity. J Am Geriatr Soc 2009;57:486-91.

37. Reed M. When the brain gets hard of hearing: paying attention to cognition in hearing rehabilitation. Seminars on Audition. Can Hear Rep 2012;8:42-5.

38. Young T, Manthorp C. Towards a code of practice for effective communication with people with dementing illnesses. J Lang Soc Psychol 2009;28:174-89.

39. Schneider JM, Gopinath B, McMahon CM, et al. Dual sensory impairment in older age. J Aging Health 2011;23:1309-24.

40. Heine C, Browning C. Dual sensory loss in older adults: a systematic review. Gerontologist 2015;55:913-28. http://dx.doi.org/10.1093/ geront/gnv074

41. Behrman S, Chouliaras L, Ebmeier KP. Considering the senses in the diagnosis and management of dementia. Maturitas 2014;77:305-10.

42. Bédard $\mathrm{E}$, Kergoat $\mathrm{H}$, Kergoat MJ, et al. Systematic review of vision-related quality of life questionnaires for older institutionalised seniors with dementia. Ophthalmic Physiological Optics 2015;35:377-87.

43. Gallacher J. Hearing, cognitive impairment and aging: a critical review. Rev Clin Gerontol 2004;14:199-209.

44. Dammeyer J. Deafblindness: a review of the literature. Scand $J$ Public Health 2014;42:554-62.

45. Hersh MA. Deafblind people, stigma and the use of communication and mobility assistive devices. Technol Disabil 2013;25: 245-61.

46. Saunders GH, Echt KV. An overview of dual sensory impairment in older adults: perspectives for rehabilitation. Trends Amplif 2007:11:243-58.

47. Arksey H, O'Malley L. Scoping studies: towards a methodological framework. Int J Soc Res Methodol 2005;8:19-32.

48. Armstrong R, Hall BJ, Doyle J, et al. 'Scoping the scope' of a Cochrane review. J Public Health 2011;33:147-50.

49. Colquhoun $\mathrm{HL}$, Levac D, O'Brien KK, et al. Scoping reviews: time for clarity in definition, methods, and reporting. J Clin Epidemiol 2014;67:1291-4.

50. Levac D, Colquhoun $\mathrm{H}$, O'Brien KK. Scoping studies: advancing the methodology. Implementation Sci 2010;5:1-9.

51. Daudt HML, van Mossel C, Scott SJ. Enhancing the scoping study methodology: a large, inter-professional team's experience with Arksey and O'Malley's framework. BMC Med Res Methodol 2013;13:48.

52. Moher D, Shamseer L, Clarke M, et al. Preferred reporting items for systematic review and meta-analysis protocols (PRISMA-P) 2015 statement. Syst Rev 2015:4:1.

53. Rowe BH, Oxman AD. An assessment of the sensibility of a quality-of-life instrument. Am J Emerg Med 1993;11:374-80.

54. Yeung E, Woods N, Dubrowski A, et al. Sensibility of a new instrument to assess clinical reasoning in post-graduate orthopaedic manual physical therapy education. Man Ther 2015;20:303-12.

55. The American Speech-Language-Hearing Association, ASHA. Hearing Screening. 2016. http://www.asha.org/public/hearing/ Hearing-Screening

56. Ferris FL III, Kassoff A, Bresnick GH, et al. New visual acuity charts for clinical research. Am J Ophthalmol 1982;94:91-6.

57. Wittich W, Sikora L, Watanabe DH, et al. Canadian research contributions to low vision rehabilitation: a quantitative systematic 
review. Canadian J Optom 2012;74:30-7. http://opto.ca/sites/default/ files/resources/documents/cjo_volume_74_no_3_canadian_ research_contributions_to_low_vision_rehabilitation.pdf

58. Squires JE, Estabrooks CA, O'Rourke HM, et al. A systematic review of the psychometric properties of self-report research utilization measures used in healthcare. Implementation Sci 2011;6:83.

59. Feinstein AR. The theory and evaluation of sensibility. In: Feinstein AR, ed. Clinimetrics. New Haven, MA: Y.U. Press, 1987:141-66.

60. Bowen DJ, Kreuter M, Spring B, et al. How we design feasibility studies. Am J Prev Med 2009;36:452-7.
61. Fitch K, Bernstein SJ, Aguilar MD, et al. The RAND/UCLA appropriateness method user's manual (No. RAND/ MR-1269-DG-XII/RE). 2001. http://www.rand.org/pubs/monograph_ reports/MR1269.html

62. Ludwig B. Predicting the future: have you considered using the Delphi methodology? J Extension 1997;35:93-6.

63. Rockwood K, Fay S, Hamilton L, et al. Good days and bad days in dementia: a qualitative chart review of variable symptom expression. Int Psychogeriatr 2014;26:1239-46.

64. Dullard B, Saunders GH. Documentation of dual sensory impairment in electronic medical records. Gerontologist 2016;56:313-17. 